\title{
Real state of the physical properties of space and time
}

\section{Boriev I.A.}

The Branch of Talrose Institute for Energy Problems of Chemical Physics of Russian Academy of Sciences, Chernogolovka, Moscow region, Russia;

E-mail: Boriev <boriev@binep.ac.ru>;

Astronomical and astrophysical data indicate the presence in surrounding space of the dark matter, which needs to explain the observed dynamics of Galaxies in the framework of Newtonian mechanics and also to solve the problems of the gravity and the stationarity of the Universe. This dark matter with its assessed very low density $\left(\sim 10^{-29} \mathrm{~g} / \mathrm{cm}^{3}\right)$ is main part of the matter in the Universe, because its mass is $\sim 10$ times greater than the mass of all visible cosmic bodies. It is clear that namely the properties of dark matter, which fills the real space, must determine the physical properties of space and, therefore, the fundamental physical laws, which take place in the nature.

Indeed, using known properties of the cosmic microwave radiation, the energy of which amounts to 90\% of total energy for all measured cosmic radiation, and using logical assumption that this radiation is produced by motion of dark matter, it can be shown that the conservation laws of classical physics and the principles of quantum mechanics receive their materialistic basis. For example, high homogeneity and isotropy $\left(\sim 10^{-4}\right)$ of this radiation indicate the same properties of dark matter and, consequently, the same properties of real space filled by this matter, what according to known mathematical theorems of E. Noether justifies the applicability of conservation laws of momentum and angular momentum, correspondingly. Moreover, the fact that this radiation has a spectrum of the black body (at temperature $\sim 2.7 \mathrm{~K}$ ) and has a maximum at the wavelength $\sim 1.9 \cdot 10^{-3} \mathrm{~m}$ allows determine the value of mechanical action produced by corresponding oscillatory motion of dark matter. The value obtained corresponds well to Planck's constant $\left(6.6 \cdot 10^{-34} \mathrm{~J} \cdot \mathrm{s}\right)$, which, in essence, is the mechanical action, what gives materialistic justification to the Heisenberg's uncertainty principle and to other concepts of quantum mechanics.

As regards to the physical meaning of the concept "time", it was introduced, as well known, by man mind to describe the dynamics of observed processes taking place in the nature. And, as it's also well known, the concept "time" is used solely for comparative description of these processes, because for that one of these processes is used as a "reference clock". So, it's not wonder that, as they say, everything is relative and everything is coming to know by comparison. Therefore, such use of the concept "time" is no doubt reasonable and effective.

Thus, as is obvious, in the nature occur a variety of processes involving matter, behavior of which corresponds well to known fundamental laws of physics (classical and quantum) since these laws are determined by observed properties of dark matter filling all space. Therefore, real space is material, being filled by dark matter, and has high homogeneity and isotropy for the large scale, but for enough small scale this material space exhibits equilibrium seesaw motion at 2.7 K. As regards the concept "time", a reasonable understanding is true, that actually in the nature there is no "time". Such understanding let solve logically the old problem of "arrow of time" (no real "time" - no problem) and also indicates that the use of four-dimensional space-time representation requires a definite accuracy, because the coordinate "time" does not really exist.

Keywords: actual properties of space, microwave background radiation, dark matter, fundamental laws of classical and quantum physics, Planck's constant, Neter's theorems, relativity principle, concept of time, arrow of time.

DOI: $10.18698 / 2309-7604-2015-1-101-111$ 


\section{Introduction}

To describe the observed phenomena in the nature two important concepts (space and time) were introduced long ago by human mind. Both they are fundamental concepts of physics, which is the basic science of human knowledge, and are used overall to investigate and explain these phenomena. For example, the main task of classical physics is to explain the observed motion of material bodies in the surrounding space, which is considered as Euclidean and is characterized by metric standard. This task has been solved using the concept of time (necessary with the use of certain reference clock), what allows to give a quantitative description of this motion.

On the basis of these two concepts, and also the concept of the mass of material body, the effective mathematical apparatus of the theoretical physics was created. These concepts with the use of experimentally determined fundamental laws of classical physics and conceptions of quantum mechanics let describe well the majority of observed phenomena in the nature. Here, it should be noted that these laws and conceptions do not yet received their vindication, based on more fundamental substantiation, but are applied confidently only because they are in good agreement with the experimental data and allow make quite accurate predictions.

Moreover in the present state of physics there are some old and, unfortunately, new issue, which need to be reasonably decided. As to new issue, sufficiently note the recently emerging idea of dark energy, which has not its reasonable explanation, and is the consequence of another strange idea of expansion, even though accelerated expansion, of the Universe. Concerning the long-used fundamental concepts of space and time there are, as known, several unsolved issue. So, remains without answer the important question: is the space totally empty (i.e. vacuum), from which, nevertheless, may appear material particles in the process of electron-positron pair creation, or the space is material, and then this process gets logical justification, at least? And how exactly real physical properties of the space fit, as supposed, to Euclidean geometry? As regards the concept of time, there are long discussed questions: is time absolute or relative, and whether it exists in reality? Thus, there are unsolved problems about real physical properties of these two fundamental concepts of physics. As clear their reasonable solution is necessary in order to create objective base of physics and to provide its further proper development.

Before solve these problems of physics it is necessary, first, to define the subject of discussion, i.e. establish that is physics as a science, and, secondly, justify the criterion, what in physics (its experimental results and theoretical concepts) should be considered as correct. No doubt, physics is the science intended primarily for experimental study of observed properties in the nature. And secondly the theoretical physics was developed for explanation of these properties 
on the basis of the concepts, entered by human mind, and with the use of established fundamental physical laws. Therefore, physics is an objective science, because it proceeds from objectivity of reliably established experimental data and from priority of such data over the methods of their theoretical explanations. Thus, the objective criterion of correctness of entered concepts in theoretical physics is their ability correctly explains the experimental data, and, in addition, gives reliable predictions of new phenomena. Therefore, entered concepts, established laws and assumptions of physics should be reasonably changed, if they do not fully describe the reliable experimental data, and lead to erroneous predictions or to any anomalies and paradoxes.

Now cumulated astronomical and astrophysical data indicate the presence in all surrounding space of so-called dark matter [1-4], whose experimental registration is now actively seeking for. The existence in the space of dark matter, which so far is considered as invisible and therefore called "dark", needs foremost to explain the observed dynamics of Galaxies in the framework of Newtonian mechanics and also for solution the problems of gravity and of the Universe stationary state. Besides, the existence of dark matter, i.e. the materiality of "vacuum", let logically explain the emergence of material particles in the processes of electron-positron pair creation and give reasonable extension of the standard model of elementary particles. According to condition of the Universe stationarity dark matter has a very low density $\left(\sim 10^{-29} \mathrm{~g} / \mathrm{cm}^{3}\right)$, but it is the main part of matter in the Universe, because its mass should be $\sim 10$ times greater than the mass of all visible cosmic bodies. No doubt, that namely the properties of dark matter, which fills the real space, must determine physical properties of the space and, therefore, all fundamental physical laws, which take place in the nature.

Indeed, using known properties of the cosmic microwave background radiation (CMBR) [5], which energy amounts to $90 \%$ of total energy for all measured cosmic radiation, and using logical assumption that this radiation is produced by corresponding motion of dark matter, it can be shown that the conservation laws of classical physics and the principles of quantum mechanics receive their materialistic basis [6,7]. For example, high homogeneity and isotropy $\left(\sim 10^{-4}\right)$ of this radiation indicate the same properties of dark matter and, consequently, the same properties of real space filled by this matter, what according to known mathematical theorems of E. Noether [8] justifies the applicability of conservation laws of momentum and angular momentum, correspondingly. Moreover, the fact that this radiation has a spectrum of the black body (at $\sim 2.7$ $\mathrm{K}$ ) and has a maximum at the wavelength $\sim 1.9 \cdot 10^{-3} \mathrm{~m} \mathrm{[5]} \mathrm{allows} \mathrm{determine} \mathrm{the} \mathrm{value} \mathrm{of} \mathrm{mechanical}$ action produced by equilibrium see-sew motion of dark matter. The value obtained [6,7] corresponds well to Planck's constant $\left(6.6 \cdot 10^{-34} \mathrm{~J} \cdot \mathrm{s}\right)$, which, in essence, is the mechanical action, 
what gives materialistic justification to the Heisenberg's uncertainty principle and to other conceptions of quantum mechanics.

As regards the physical meaning of the concept "time", it was introduced, as well known, by human mind to describe the dynamics of observed processes taking place in the nature. And, as it's also well known, the concept "time" is used solely for comparative description of these processes, because for that one of these processes is used as a "reference clock". So, it's not wonder that, as they say, everything is relative and everything is coming to know by comparison. Therefore, such use of the concept "time" is no doubt reasonable and effective, though it is not the "time" in reality.

Thus, as is obvious, in the nature ever occur a variety of processes involving matter, behavior of which corresponds well to known fundamental laws of physics (classical and quantum) since these laws are determined by observed properties of dark matter filling all space. Therefore, real space is material, being filled by dark matter, and has high homogeneity and isotropy for the large scale, but for enough small scale this material space exhibits equilibrium seesaw motion with amplitude $\sim 1.9 \cdot 10^{-3} \mathrm{~m}$ and at dark matter temperature $\sim 2.7 \mathrm{~K}$. As to the concept "time", a reasonable understanding is true, that actually in the nature there is no "time", since this concept was reasonably created only by human mind. Such understanding let solve logically the old problem of "arrow of time" (no real "time" - no problem) and also indicates that the use of fourdimensional space-time representation requires a definite accuracy, because the coordinate "time" does not really exist.

\section{Dark matter properties define fundamental laws both classical and quantum physics}

Used fundamental laws of classical physics (conservation laws of momentum, angular momentum and energy) and also the principles of quantum mechanics (Planck`s constant, Heisenberg`s uncertainty principle, de Broglie`s wave mechanics, etc) have so far no physical (materialistic) substantiation of their origin. They are not derived from any higher order laws or principles however they are used with confidence because all they are in good agreement with obtained experimental data. Really, they do not contradict to known experimental data and are not refused by them, but only in the case if they are used in corresponding very different domain of physics: classical or quantum. Last circumstance means a strange splitting of the physics and created some hardly explainable problems, specifically the concept of corpuscular-wave dualism. However, no doubt, that all known fundamental laws of classical physics and also the principles of quantum mechanics must have their joint materialistic substantiation on the base of the real 
properties of the space filled by dark matter. Indeed, the existence in space of dark matter and established properties of CMBR at reasonable assumption, that CMBR is produced by certain motion of dark matter, allow to reveal the materialistic base for fundamental conservation laws of classical physics and the reason for all principles of quantum mechanics [6,7].

Thus, high spatial homogeneity and isotropy of CMBR and, naturally, the same properties of its producing dark matter, validate conservation laws of momentum and angular momentum, correspondingly, in accord with known E. Noether`s theorems (1918) [8]. These mathematical theorems assert that to each conservation law of physics match up the certain property of space, particularly, conservation laws of momentum and angular momentum will hold true only if infinite space is, correspondingly, highly homogeneous and highly isotropic.

Besides, CMBR properties (blackbody spectrum at temperature $\mathrm{T} \sim 2.7 \mathrm{~K}$ with maximum wavelength $\lambda \approx 1.9 \cdot 10^{-3} \mathrm{~m}$ ) [5] let obtain the kinetic energy of producing this spectrum the equilibrium seesaw motion of dark matter (with the use of the Boltzmann`s constant). From the energy of such motion (certainly with the speed of electromagnetic waves $c=3 \cdot 10^{8} \mathrm{~m} / \mathrm{s}$ ) the value of action function for seesaw motion of dark matter may be received. The obtained value of this action function $\left(\sim 7 \cdot 10^{-34} \mathrm{~J} \cdot \mathrm{s}\right)[6,7]$ is well equal to Planck`s constant $\mathrm{h}=6.6 \cdot 10^{-34} \mathrm{~J} \cdot \mathrm{s}$, which really is an action function too. This result discovers materialistic origin of Planck`s constant due to such seesaw motion of dark matter, and gives materialistic base to Heisenberg`s principle of uncertainty, which follows from the existence of Planck`s constant, and also to all others conceptions of quantum mechanics.

As clear, such dark matter seesaw motion must strong disturb, as observed, the spatial motion of light elementary particles (like electron), what may be correctly described by the methods of quantum wave mechanics, which was proposed by L. de Broglie. But this dark matter seesaw motion could not significantly disturb spatial motion of much heavier particles (atoms, molecules and so on), therefore their observed spatial motion may be described well by fundamental laws of classical physics. Thus, dark matter seesaw motion, producing the CMBR, let logically explain the reason and the necessity of different (classical or quantum) description of physical processes in the nature.

Besides, seesaw motion of dark matter also explains the reason of the physical essence of corpuscular-wave dualism. Simply speaking, spatial motion of light elementary particles (like electron, etc) should undergo the strong disturbing influence from dark matter seesaw motion. Such disturbed motion of these particles may be described well by methods of quantum wave mechanics taking into account an action function value of dark matter (equal to Planck`s constant). 
But, as clear, when such elementary particles interact (encounter) with another particles, they act as a corpuscular, which they really are.

As known from 1918, E. Noether's mathematical theorems [8] denote conditions for realization of three fundamental conservation laws of classical physics. Thus, momentum conservation law takes place if space is uniform, angular momentum conservation law takes place if space is isotropic, and energy conservation law takes place if the trend of physical processes is independent on its starting moment. As clear, these laws could really take place in the nature if the space obeys the properties stated by E. Noether`s theorems. At present, the applicability of classical physics conservation laws is based on assumption that space is empty and possess the properties of Euclidian geometry (i.e. uniform and isotropic), what accord to conditions of E. Noether`s theorems. However, resent cosmological data indicate that real space is not empty, but is filled by actively sought-for dark matter, experimental visualization of which is now the main problem of cosmology [1-4]. As it was earlier noted briefly at the first time [7], namely the properties of dark matter and CMBR, produced by it, must determine all fundamental laws as classical physics and quantum mechanics.

As discovered in 1965 by Arno Pensias and Robert Wilson, the observed from the Earth a weak enough (like a noise) cosmic microwave radiation are characterized by high isotropy and a temperature about $\sim 3 \mathrm{~K}$ (for that they received Nobel Prize in physics in 1978). Now it is well established, that this radiation, which is known as CMBR, is the most powerful among the all detected radiations from the space (about $90 \%$ of their total energy) and is characterized by very high isotropy and also by high homogeneity. It is clear that observed high isotropy and high homogeneity of CMBR mean that producing it dark matter has the same properties (in large space scales). Hence the ambient space, filled by dark matter, is also highly isotropic and highly uniform, what according to conditions of E. Noether`s theorems give real materialistic base for realization in the nature of the fundamental laws of angular momentum and momentum conservation, correspondingly.

As to fundamental law of energy conservation, it takes place, according to E. Noether`s theorem, if the run of observed physical processes is not depend on its starting moment, what means "time" constancy (or uniform flow of "time"). Such condition is well consistent with observed independence of behavior (on starting moment) of all physical processes in our materialistic nature. In this connection it should be understand that the mankind always use for metering the "time" flow some other physical process, which is used as a "reference clock". 
As a resulting important conclusion, it must be underlined that fundamental conservation laws of classical physics operate well in the nature not because of the E. Noether's theorems exist by itself, but because of the real properties of ambient space, which is filled by dark matter, are in accord with conditions of E. Noether`s theorems.

\section{Planck's constant value follows from the properties of dark matter see-sew motion}

As well established observed CMBR has the blackbody spectrum at temperature $\sim 2.7 \mathrm{~K}$ with maximum wavelength $\sim 1.9 \cdot 10^{-3} \mathrm{~m}$. Taking into account the existence of dark matter, which fills all ambient space and determines dynamics of astrophysical bodies in the Universe, it is quite reasonable assume that CMBR is produced by certain motion of this dark matter. Such materialistic point of view, which, as shown above, let substantiate the applicability of fundamental laws of classical physics, no doubt must also reveal the materialistic reason of quantum mechanics and its principles, including known value of Planck`s constant.

The fact, that CMBR has blackbody spectrum at $\mathrm{T} \approx 2.7 \mathrm{~K}$, demonstrates that dark matter, which creates CMBR, is in equilibrium seesaw motion at this temperature. Taking into account that dark matter is really in equilibrium motion, the value of kinetic energy of dark matter seesaw motion $(\varepsilon)$ should be equal to dark matter thermal energy, which may be obtained from dark matter temperature with the use of the universal Boltzmann`s constant $\mathrm{k}=1.38 \cdot 10^{-23} \mathrm{~J} / \mathrm{K}$. As known, Boltzmann`s constant let connect the temperature of equilibrium thermal motion of some parts of any matter with the mean-square velocity of kinetic motion of these parts. In the case of dark matter seesaw motion, the velocity of this motion should be equal to the known electromagnetic waves speed $\left(\mathrm{c}=3 \cdot 10^{8} \mathrm{~m} / \mathrm{s}\right)$ since these waves propagate namely in dark matter filling all ambient space. So, the value of dark matter kinetic energy $\varepsilon$ should be equal to its thermal energy: $\varepsilon=3 \mathrm{kT} / 2=5.6 \cdot 10^{-23} \mathrm{~J}$. This result let estimate the mean mass of parts of dark matter, namely, its effective mass (m), which produces such seesaw motion of dark matter:

$$
m=2 e / c^{2} \sim 1.2 \times 10^{-39} \mathrm{~kg}
$$

It is clear that observed maximum wavelength of $\operatorname{CMBR}\left(\lambda \approx 1.9 \cdot 10^{-3} \mathrm{~m}\right)$ is well equal to the mean value of the amplitude of dark matter seesaw motion, and that this amplitude corresponds to the damping length of dark matter seesaw motion. It is also clear, that $\lambda$ means the damping 
length for momentum (p) of dark matter effective mass seesaw motion, and this momentum is equal to $\mathrm{p}=\mathrm{m} \cdot \mathrm{c}$.

The established parameters of dark matter seesaw motion (the effective mass $\mathrm{m}$, its motion speed $\mathrm{c}$ and the damping length $\lambda$ of $\mathrm{p}$ ) let estimate the value of mechanical action (an action function) for such dark matter seesaw motion by two known manner, which are used in classical mechanics and in quantum mechanics.

According to conception of classical mechanics an action function value (S) is a measure of physical motion and is equal to the product of energy and a "time" of this energy existence (dissipation). Taking into account that full circle path of dark matter seesaw motion energy is equal to $2 \lambda$, its "time" is equal to $2 \lambda / c \approx 1.3 \cdot 10^{-11} \mathrm{~s}$. So, corresponding value of $\mathrm{S}$ for dark matter seesaw motion is equal to

$$
S=2 e l / c \approx 5.6 \times 10^{-23} \times 1.3 \times 10^{-11} J \times S \approx 7.3 \times 10^{-34} J \times S
$$

As it is seen, such classically obtained estimation of the $S$ value for an action function of dark matter seesaw motion corresponds well to the Planck`s constant $\mathrm{h}$, which physically is also an action function.

As to quantum mechanics, the $S$ value usually is obtained by multiplying the mechanical momentum and its damping length. In the case of dark matter seesaw motion its mechanical momentum is equal to $\mathrm{p}=\mathrm{m} \cdot \mathrm{c}$ and damping length of this momentum is equal to $\lambda$. So, according to the manner of quantum mechanics, an action function of dark matter seesaw motion should be equal to multiplication $\mathrm{p}$ and $\lambda$ :

$$
S=m \times c \times I \approx 1.2 \times 10^{-39} \times 3 \times 10^{8} \times 1.9 \times 10^{-3} \approx 6.8 \times 10^{-34} J \times S .
$$

As is obvious, such obtained estimation of the $S$ value for an action function of dark matter seesaw motion also corresponds well to Planck`s constant h.

It is clear that these two estimations of $S$ discover the origin of Planck`s constant $h$ from dark matter seesaw motion, namely at dark matter temperature $\sim 2.7 \mathrm{~K}$. No doubt, what important to note, that if dark matter temperature (or any other dark matter parameter) will change then it will course the change of dark matter action function and, correspondingly, the change of the value of Planck`s constant h. No doubt, the obtained result for h origin from dark matter seesaw motion 
gives materialistic base to the Heisenberg`s principle of uncertainty and to all other conceptions of quantum mechanics.

It should be underlined, that revealed dark matter seesaw motion gives full vindication of the statistical physics adaptability. Really, the dark matter seesaw motion (at temperature $\sim 2.7 \mathrm{~K}$ ) means, like to known thermal Brownian motion, that the trajectories of all material bodies in the space should be entangled in some extend and, correspondingly, they may be intersected by itself. Such real property of body trajectory rejects the one of the main postulate of classical mechanics (about a non-crossing trajectory of any body), and prove the truth of Boltzmann`s approach in statistical physics.

\section{Real physical meaning of the concept "time"}

The concept "time" was introduced by human mind to describe the dynamics of observed processes taking place in the nature. And, as well known, the concept "time" is used solely for comparative description of these processes, because for that one of these processes is used as a "reference clock". In its long history mankind has used as a "reference clock" many very different materialistic processes taking place in the nature. Now as a "reference clock" is used an "atomic clock", which is based on the frequency of atomic radiation (that also means the use of real materialistic process).

Therefore, it is clear, that kinetic properties of all physical processes taking place in the nature come to know by mankind due to their comparison with any other physical (materialistic) process. No doubt, such understanding means that really in the nature there is no "time": neither absolute "time" no relative "time". The overall using by mankind of the concept "time" is simply a very convenient parametrical approach to describe observed kinetics of physical processes in our materialistic nature (no more, than that).

Such understanding of the concept "time" let solve logically the old problem of "arrow of time" (no real "time" - no problem) and also indicates that the use of four-dimensional space-time representation requires a definite accuracy, because the coordinate "time" does not really exist.

\section{Conclusion}

Real physical properties of space are determined by observed properties of CMBR [5], which are produced by corresponding seesaw motion of dark matter, which fills, in accord to resent date [1-4], all ambient space. Such reasonable understanding of materialistic sense of space let substantiate, on the base of CMBR features, as fundamental conservation laws of classical physics 
and also simple materialistic origin of principles of quantum mechanics [6,7]. No doubt, these results finally vindicate the existence of dark matter in ambient space. According to CMBR features the space, filled by dark matter, is at large scales highly homogeneous and isotropic, what is in accord with Euclidean geometry, but at enough small scales the dark matter of the space is in equilibrium seesaw motion at $\sim 2.7 \mathrm{~K}$ with amplitude $\sim 1.9 \cdot 10^{-3} \mathrm{~m}$, what creates the value of the (mechanical) action function equal to the value of Planck`s constant $h$.

In addition, it is necessary underline that the observed (from the Earth) weak $(\sim 0.1 \%)$ dipole anisotropy of CMBR [5] also directly indicates the existence in space of some material substance, through which the Solar system moves (at the rate $\sim 400 \mathrm{~km} / \mathrm{s}$ ) in direction of the Leo constellation. This feature of CMBR is very important since it directly demonstrates that the "vacuum" is not empty, but is filled by some substance, namely, by actively sought-for dark matter. As regards the resent problem of cosmology, namely, the experimental registration of any properties of dark matter, which is considered as substance neither emitting, no absorbing electromagnetic radiation [1-4], it is clear, that the observed CMBR is real experimental display of dark matter.

The very important conclusion is that the existence of dark matter in the space let logically explain the observed redshift for electromagnetic waves, coming from remote regions of the Universe, by reasonable energy dissipation of these waves at their passage through material substance (namely dark matter), what obviously should lead to increase of their wavelengths. At that, as clear, this increase should be larger for waves coming from larger distance, as it is really observed. Such reasonable understanding let remove the very strange assumption of Universe expansion, and also the accelerated expansion, which were unconfirmed by recent (2013) Planck space vehicle mission, and which lead to more strange and totally unexplainable conception of dark energy.

As regards the physical meaning of the concept "time", it should be realized that kinetic properties of all physical processes, which take place in the nature, come to know by mankind only due to their comparison with any other physical (materialistic) process used as a "reference clock". Therefore, a reasonable understanding is true, that really in the nature there is no "time": neither absolute, no relative. Such understanding let solve logically the old problem of "arrow of time" (no real "time" - no problem), and it also indicates that the use of four-dimensional space-time representation requires a definite accuracy, because the coordinate "time" does not really exist. Nevertheless, the use of the concept "time" is very effective and convenient for description the kinetics of observed physical processes, as it's clear from the history of physics development. 
In conclusion it worth to note, that stated physical properties of space and time really exist since they let to give reasonable materialistic substantiation to fundamental laws of classical physics and to conceptions of quantum mechanics, and besides let to solve some known problems of physics.

\section{References}

1. Vanderburgh W.L. (2014). On the interpretive role of theories of gravity and "ugly" solutions to the total evidence for dark matter. Studies in History and Philosophy of Modern Physics, Vol. $44,62-67$.

2. Davini S. (2014). Enter the darkside. Nuclear Insruments and Methods in Physics Research, Vol. 742, 183-186.

3. Kosso P. (2013). Evidence of dark matter, and the interpretive role of general relativity. Studies in History and Philosophy of Modern Physics, Vol. 44, 143-147.

4. Paolo L. (2012). Open problems in particle astrophysics. Nuclear Insruments and Methods in Physics Research, Vol. 692, 106-119.

5. Tests of Big Bang (n.d.). Retrived from: http://map.gsfc.nasa.gov/universe/bb_tests_cmb.html

6. Boriev I.A. (2014). Fundamental laws of classical and quantum physics follow from the features of microwave background radiation produced by dark matter seesaw motion. International Journal of Astronomy, Astrophysics and Space Science, Vol. 2, 2, 7-11.

7. Boriev I.A. (2013). Fundamental laws of classic and quantum physics as consequence of materialistic insight into the properties of microwave background radiation. ScientificCoordination Session "Non-ideal Plasma Physics", Moscow.

8. Noether E. (1918). Invariante Varlationsprobleme. Nachr. d. König. Gesellsch. d. Wiss. Zu Göttingen, Math-phys. Klasse, 235-257. 